TRANSACTIONS OF THE

AMERICAN MATHEMATICAL SOCIETY

Volume 349, Number 10, October 1997, Pages 4007-4019

S $0002-9947(97) 01751-0$

\title{
FOUR-MANIFOLDS WITH SURFACE FUNDAMENTAL GROUPS
}

\author{
ALBERTO CAVICCHIOLI, FRIEDRICH HEGENBARTH, AND DUŠAN REPOVŠ
}

\begin{abstract}
We study the homotopy type of closed connected topological 4manifolds whose fundamental group is that of an aspherical surface $F$. Then we use surgery theory to show that these manifolds are $s$-cobordant to connected sums of simply-connected manifolds with an $\mathbb{S}^{2}$-bundle over $F$.
\end{abstract}

\section{INTRODUCTION}

In this paper we shall study closed connected oriented topological 4-manifolds $M^{4}$ such that $\Pi_{1}(M) \cong \Pi_{1}(F)$, where $F$ is a closed oriented aspherical surface, i.e. $F=K\left(\Pi_{1}, 1\right)=B \Pi_{1}$. The easiest examples of such manifolds are connected sums of the type $E \# M^{\prime}$, where $E \rightarrow F$ is an $\mathbb{S}^{2}$-bundle over $F$ and $M^{\prime}$ is a simply-connected 4-manifold. There are reasons to conjecture that any such manifold is topologically homeomorphic to some $E \# M^{\prime}$. Other natural examples of 4-manifolds with surface fundamental groups are given by certain elliptic surfaces as communicated to us by Matsumoto in [9]. Recall that a compact complex manifold of complex dimension two is said to be an elliptic surface if it is fibered over a Riemann surface with general fiber an elliptic curve, i.e. a 2 -torus $T^{2} \cong \mathbb{S}^{1} \times \mathbb{S}^{1}$. It may admit certain (possibly multiple) singular fibers (for details see [10]). It was proved in [10] that an elliptic surface is a 4-manifold whose fundamental group is isomorphic to that of a closed surface if it has positive Euler number and does not have multiple fibers (see [10], Remark 2, p. 563).

For simplicity, we will assume that $M$ is a spin manifold, i.e. $w_{2}(M)=0$, where $w_{2}$ denotes the second Stiefel-Whitney class. As a consequence, the sphere-bundle $E$ will be trivial. However, a condition weaker than $w_{2}(M)=0$ would suffice to prove Theorem 1.1 below; in fact, $w_{2}(u)=0$ is sufficient. Here $u \in H_{2}(M ; \mathbb{Z})$ is defined in Section 2.

The referee suggested that we treat also the case $w_{2}(u) \neq 0$. The proof is similar to that of Theorem 1.1, but for technical reasons we will give it in the appendix.

In Section 2 we define a map of degree $1, \psi: M \rightarrow F \times \mathbb{S}^{2}$, which gives rise to the split exact sequence

$$
0 \rightarrow K_{2}(\psi, \Lambda) \rightarrow H_{2}(M ; \Lambda) \rightarrow H_{2}\left(F \times \mathbb{S}^{2} ; \Lambda\right) \rightarrow 0
$$

Received by the editors January 20, 1995 and, in revised form, February 6, 1996.

1991 Mathematics Subject Classification. Primary 57N65, 57R67, 57Q10.

Key words and phrases. Four-manifolds, homotopy type, s-cobordism, surgery, surface fundamental groups.

Work performed under the auspices of the G.N.S.A.G.A. of the C.N.R. (National Research Council) of Italy and partially supported by the Ministero per la Ricerca Scientifica e Tecnologica of Italy within the projects "Geometria Reale e Complessa" and "Topologia" and by the Ministry for Science and Technology of the Republic of Slovenia Research Grant No. P1-0214-101-94.

(c)1997 American Mathematical Society 
where $\Lambda=\mathbb{Z}\left[\Pi_{1}(M)\right]$ is the integral group ring.

Similarly, there is a split exact sequence

$$
0 \rightarrow K_{2}(\psi, \mathbb{Z}) \rightarrow H_{2}(M ; \mathbb{Z}) \rightarrow H_{2}\left(F \times \mathbb{S}^{2} ; \mathbb{Z}\right) \rightarrow 0
$$

The splittings respect the intersection pairings. By the result of M. Freedman (see [4] and [5]) the induced intersection form on $K_{2}(\psi, \mathbb{Z})$ can be realized as intersection form of a closed simply-connected topological 4-manifold $M^{\prime}$. Let $M_{1}$ denote the connected sum of $F \times \mathbb{S}^{2}$ and $M^{\prime}$, and let

$$
c: M_{1}=\left(F \times \mathbb{S}^{2}\right) \# M^{\prime} \rightarrow F \times \mathbb{S}^{2}
$$

be the collapsing map. Since $c$ is of degree 1, we have short split exact sequences as above; in particular,

$$
0 \rightarrow K_{2}(c, \Lambda) \rightarrow H_{2}\left(M_{1} ; \Lambda\right) \rightarrow H_{2}\left(F \times \mathbb{S}^{2} ; \Lambda\right) \rightarrow 0
$$

In Section 2 we are going to construct a map from the 3-skeleton of $M_{1}$ into $M$. Furthermore, we prove that it can be extended over $M_{1}$ if the $\Lambda$-interesection forms on $K_{2}(\psi, \Lambda)$ and on $K_{2}(c, \Lambda)$ coincide.

More precisely, we have

Theorem 1.1. Let $M^{4}$ be a closed connected oriented TOP 4-manifold with $w_{2}(M)$ $=0$ and $\Pi_{1}(M) \cong \Pi_{1}(F)$, where $F$ is a closed aspherical surface. Then $M$ is simple homotopy equivalent to the connected sum $M_{1}=\left(F \times \mathbb{S}^{2}\right) \# M^{\prime}$ if and only if the $\Lambda$-intersection forms on $K_{2}(\psi, \Lambda)$ and on $K_{2}(c, \Lambda)$ are isomorphic.

In particular, if $\chi(M)=2 \chi(F)$, then $K_{2}(\psi, \Lambda) \cong 0$, hence $M$ is simple homotopy equivalent to $F \times \mathbb{S}^{2}$.

We observe that in our case any homotopy equivalence is simple because the Whitehead group of $\Pi_{1}(F)$ vanishes (see [11]). Furthermore, the manifold $M^{\prime}$ is unique, up to TOP homeomorphism, because its intersection form over $\mathbb{Z}$ must be even (see for example [5]). We also note that the second part of the statement in Theorem 1.1 gives a simple alternative proof of Theorem 3 of [6].

Using recent results of Hillman ([6], [7]) and of Cochran and Habegger ([3]), we also prove that the homotopy type classifies our manifolds, up to TOP $s$-cobordism.

Theorem 1.2. With the above notation, if $M$ is simple homotopy equivalent to $E \# M^{\prime}$, then $M$ and $E \# M^{\prime}$ are topologically s-cobordant.

The assertion was first proved for the case when $M$ is simple homotopy equivalent to $E$ by Hillman (see [6]). We also note that TOP $s$-cobordant 4-manifolds $M$ and $N$ are stably homeomorphic (see for example [5]), i.e. $M \# k\left(\mathbb{S}^{2} \times \mathbb{S}^{2}\right)$ is TOP homeomorphic to $N \# \ell\left(\mathbb{S}^{2} \times \mathbb{S}^{2}\right)$ for some integers $k, \ell \geq 0$. Thus Theorem 1.2 extends a well-known result of Wall (see [12]) to the non-simply-connected case.

In a particular case, i.e. $\Pi_{1}(M) \cong \mathbb{Z} \oplus \mathbb{Z}$, the fact that the fundamental group is elementary amenable implies that $s$-cobordisms are always topologically products (see [5]). Thus we have the following characterization result.

Theorem 1.3. Let $M^{4}$ be a closed connected oriented TOP 4-manifold with $\Pi_{1}(M)$ $\cong \mathbb{Z} \oplus \mathbb{Z}$. Let $M^{\prime}$ be the simply-connected 4-manifold defined in the discussion preceding the statement of Theorem 1.1. Then $M$ is TOP homeomorphic to the connected sum of $M^{\prime}$ with an $\mathbb{S}^{2}$-bundle over the torus if and only if the homological condition of Theorem 1.1 holds.

If further $\chi(M)=0$, then $K_{2}(\psi, \Lambda) \cong 0$, hence $M$ is homeomorphic to an $\mathbb{S}^{2}$ bundle over the torus. 
Although we work in the topological category, we occasionally use "transversality" and "regular values". This is possible by for example [5]. Moreover, we assume that $M$ has a $\mathrm{CW}$-structure. For a general reference on combinatorial homotopy of 4-complexes see [1]. For surgery theory we refer to [2] and [13].

\section{2. Номотору туре}

Let $M^{4}$ be a manifold with the properties described in Section 1 . Since $F$ is an aspherical closed surface, we have that $F=K\left(\Pi_{1}(F), 1\right)=B \Pi_{1}(F)$. For the proof of Theorem 1.1 it will not be important which isomorphism $\Pi_{1}(M) \cong \Pi_{1}(F)$ one chooses. This isomorphism is realized by a classifying map $f: M \rightarrow F$, i.e. $f$ classifies the universal covering $\widetilde{M}$ of $M$.

Lemma 2.1. There exists a map $j: F \rightarrow M$ such that the composition

$$
f \circ j: F \rightarrow F
$$

is homotopic to the identity.

Proof. There is an embedding $j_{0}: F \backslash \stackrel{\circ}{D^{2}} \simeq \bigvee_{2 g} \mathbb{S}^{1} \rightarrow M$ such that $f \circ j_{0}$ is homotopic to the inclusion $F \backslash \stackrel{\circ}{D^{2}} \rightarrow F$. Here $g$ denotes the genus of $F$. The obstruction to extending $j_{0}$ is the homotopy class $\left[\left.j_{0}\right|_{\partial D^{2}}\right] \in \Pi_{1}(M)$, and it is mapped to the obstruction to extending $f \circ j_{0}$ via the isomorphism $f_{*}: \Pi_{1}(M) \cong \Pi_{1}(F)$; hence it must be zero. Therefore $j_{0}$ extends to a map $j: F \rightarrow M$. It is now easy to see that $\operatorname{deg}(f \circ j)=1$; hence $f \circ j$ is homotopic to the identity map of $F$.

We define two elements of $H_{2}(M)$, by setting $u=j_{*}[F]$ and $v=\left[F^{\prime}\right]$, where $[F] \in H_{2}(F)$ is the fundamental class of $F, F^{\prime}=f^{-1}\left(x_{0}\right)$ and $x_{0} \in F$ is a regular value of $f$.

Lemma 2.2. The homology classes $u, v \in H_{2}(M)$ have the following intersection numbers:

(1) $u \circ v=1$; and

(2) $v \circ v=0$.

Proof. (1) Let PD : $H^{2}(M) \rightarrow H_{2}(M)$ denote the Poincaré duality isomorphism and let $\omega_{F} \in H^{2}(F)$ be the dual class of $[F]$. Then we have that

$$
\mathrm{PD}^{-1}(v)=\mathrm{PD}^{-1}\left[F^{\prime}\right]=f^{*}\left(\omega_{F}\right) .
$$

So we obtain that

$$
\begin{aligned}
u \circ v=\left(\mathrm{PD}^{-1}(u) \cup \mathrm{PD}^{-1}(v)\right) \cap[M] & =\mathrm{PD}^{-1}(v) \cap j_{*}[F]=f^{*}\left(\omega_{F}\right) \cap j_{*}[F] \\
& =j^{*} \circ f^{*}\left(\omega_{F}\right) \cap[F]=1,
\end{aligned}
$$

since $j^{*} \circ f^{*}=(f \circ j)^{*}=$ identity.

(2) Choosing a regular value $x_{0}^{\prime}$ near to $x_{0}$ yields $\left[f^{-1}\left(x_{0}^{\prime}\right)\right]=\left[f^{-1}\left(x_{0}\right)\right]=v$. But obviously, $f^{-1}\left(x_{0}^{\prime}\right) \cap f^{-1}\left(x_{0}\right)$ is empty, hence $v \circ v=0$.

Set $a=u \circ u$. The intersection matrix of the pair $(u, v)$ is

$$
\left(\begin{array}{ll}
a & 1 \\
1 & 0
\end{array}\right)
$$


The hypothesis $w_{2}(M)=0$ implies that $a \equiv 0(\bmod 2)$, i.e. $a=2 k$, for some integer $k$. The change $u \rightarrow u-k v$ produces the intersection matrix

$$
\left(\begin{array}{ll}
0 & 1 \\
1 & 0
\end{array}\right)
$$

Lemma 2.3. There exists a map $j^{\prime}: F \rightarrow M$ with the following properties:

(1) $f \circ j^{\prime}$ is homotopic to the identity; and

(2) $j_{*}^{\prime}[F]=u-k v$.

Proof. First, we represent the homology class $v=\left[F^{\prime}\right]$ by an immersed 2-sphere $\varphi: \mathbb{S}^{2} \rightarrow M$. We choose a collection of embedded circles in $F^{\prime}$ whose homology classes form a symplectic basis for $H_{1}\left(F^{\prime}\right)$. Then from this basis we choose a single generator for each handle of $F^{\prime}$. Next, we note that $\Pi_{1}\left(F^{\prime}\right) \rightarrow \Pi_{1}(M)$ is the trivial homomorphism. Therefore, by the general position each of the chosen circles bounds a 2-disc immersed into $M$ (see [5]). We use these immersed discs to surger $F^{\prime}$ and the result is an immersed sphere $\Sigma^{2}$ which represents the homology class $v$. Then $j(F) \# k\left(-\Sigma^{2}\right)$ is the image of a map $j^{\prime}: F \rightarrow M$ which satisfies properties (1) and (2) of the statement. If $\varphi: \mathbb{S}^{2} \rightarrow M$ represents the immersed 2-sphere $\Sigma^{2} \subset M$, we have $j^{\prime}=j \# k \varphi$ as required.

Remark. Obviously, we can always assume that the map $j: F \rightarrow M$ is an immersion. Thus $\Sigma^{2} \subset M$ is an algebraic dual of $j(F)$.

From now on we shall assume that $j: F \rightarrow M$ is already chosen so that it satisfies the properties of the following corollary.

Corollary 2.4. There is a map $j: F \rightarrow M$ such that:

(1) $f \circ j$ is homotopic to the identity; and

(2) the intersection matrix of the pair $u=j_{*}[F], v=\left[F^{\prime}\right]$ is

$$
\left(\begin{array}{ll}
0 & 1 \\
1 & 0
\end{array}\right) \text { if } w_{2}(u)=0 \quad \text { and } \quad\left(\begin{array}{ll}
1 & 1 \\
1 & 0
\end{array}\right) \text { if } w_{2}(u)=1
$$

Recall that $\mathrm{PD}^{-1}(v)=f^{*}\left(\omega_{F}\right)$ and $f_{*}(u)=[F]$. The next goal is to construct a map $g: M \rightarrow \mathbb{S}^{2}$ such that $g_{*}(v)=\left[\mathbb{S}^{2}\right]$ generates $H_{2}\left(\mathbb{S}^{2}\right)$. But the property $g_{*}(v)=\left[\mathbb{S}^{2}\right]$ follows from the relation $g^{*}\left(\omega_{\mathbb{S}^{2}}\right)=\mathrm{PD}^{-1}(u)$, where $\omega_{\mathbb{S}^{2}} \in H^{2}\left(\mathbb{S}^{2}\right)$ is the dual of $\left[\mathbb{S}^{2}\right]$. This holds because

$$
\begin{aligned}
1=u \circ v=\left(\mathrm{PD}^{-1}(u) \cup \mathrm{PD}^{-1}(v)\right) \cap[M] & =\mathrm{PD}^{-1}(u) \cap v=g^{*}\left(\omega_{\mathbb{S}^{2}}\right) \cap v \\
& =g_{*}\left(g^{*}\left(\omega_{\mathbb{S}^{2}}\right) \cap v\right)=\omega_{\mathbb{S}^{2}} \cap g_{*}(v),
\end{aligned}
$$

i.e. $g_{*}(v)=\left[\mathbb{S}^{2}\right]\left(\right.$ note that $g^{*}\left(\omega_{\mathbb{S}^{2}}\right) \cap v \in H_{0}(M)$ and $\left.g_{*}=\mathrm{Id}: H_{0}(M) \rightarrow H_{0}\left(\mathbb{S}^{2}\right)\right)$.

Lemma 2.5. There exists a map $g: M \rightarrow \mathbb{S}^{2}$ such that $g^{*}\left(\omega_{\mathbb{S}^{2}}\right)=\mathrm{PD}^{-1}(u)$, where $\omega_{\mathbb{S}^{2}}$ is the generator of $H^{2}\left(\mathbb{S}^{2}\right)$.

Proof. Let $g^{\prime}: M \rightarrow K(\mathbb{Z}, 2)=\mathbb{C} P^{\infty}$ be a map which represents the cohomology class $\mathrm{PD}^{-1}(u) \in H^{2}(M) \cong[M, K(\mathbb{Z}, 2)]$. Since $M$ has dimension four, we can assume $g^{\prime}: M \rightarrow \mathbb{C} P^{2}=\mathbb{C} P^{1} \cup_{\eta} D^{4}$, where $\eta: \mathbb{S}^{3} \rightarrow \mathbb{C} P^{1}=\mathbb{S}^{2}$ is the Hopf map. Now $\mathrm{PD}^{-1}\left(u^{2}\right)=a \omega_{M}=0$, where $\omega_{M}$ is the dual of the fundamental class of $M$. Thus $g^{\prime}$ factors over $g: M \rightarrow \mathbb{C} P^{1}=\mathbb{S}^{2}$.

Note that the map $\psi=f \times g: M \rightarrow F \times \mathbb{S}^{2}$ has degree one. We use this map to prove the following result. 
Proposition 2.6. There exists a map $\alpha: F \times \mathbb{S}^{2} \backslash \stackrel{\circ}{D^{4}} \rightarrow M$ such that $\psi \circ \alpha$ is homotopic to the inclusion $F \times \mathbb{S}^{2} \backslash \stackrel{\circ}{D^{4}} \rightarrow F \times \mathbb{S}^{2}$.

Proof. Recall that we have constructed $j: F \rightarrow M$ and $\varphi: \mathbb{S}^{2} \rightarrow M$, i.e. we have a map $j \vee \varphi: F \vee \mathbb{S}^{2} \rightarrow M$. The first obstruction to extending $j \vee \varphi$ to $F \times \mathbb{S}^{2}$ lies in the cohomology group $H^{3}\left(F \times \mathbb{S}^{2} ; \Pi_{2}(M)\right)$ with local coefficients. Poincaré duality now implies that $H^{3}\left(F \times \mathbb{S}^{2} ; \Pi_{2}(M)\right) \cong H_{1}\left(F \times \mathbb{S}^{2} ; \Pi_{2}(M)\right)$. By a result of Hillman (see [6], p. 279), one has that

$$
\begin{aligned}
\Pi_{2}(M) \cong H_{2}(M ; \Lambda) & \cong \operatorname{Ext}_{\Lambda}^{2}\left(H_{0}(M ; \Lambda), \Lambda\right) \oplus \operatorname{Ext}_{\Lambda}^{0}\left(H_{2}(M ; \Lambda), \Lambda\right) \\
& \cong H^{2}(F) \oplus \operatorname{Ext}_{\Lambda}^{0}\left(H_{2}(M ; \Lambda), \Lambda\right),
\end{aligned}
$$

where the $\Lambda$-module $Q=\operatorname{Ext}_{\Lambda}^{0}\left(H_{2}(M ; \Lambda), \Lambda\right)$ is stably $\Lambda$-free. Here $\Lambda$ is as usual the group ring $\mathbb{Z}\left[\Pi_{1}(M)\right]$. The fact that $\operatorname{Ker}\left(\psi_{*}: H_{2}(M ; \Lambda) \rightarrow H_{2}\left(F \times \mathbb{S}^{2} ; \Lambda\right)\right)$ is stably $\Lambda$-free follows from [13]. Since $Q$ is stably $\Lambda$-free, we have

$$
H_{1}\left(F \times \mathbb{S}^{2} ; Q\right) \cong \operatorname{Tor}_{1}^{\Lambda}(\mathbb{Z}, Q) \cong 0
$$

Hence we obtain

$$
H_{1}\left(F \times \mathbb{S}^{2} ; \Pi_{2}(M)\right) \cong H_{1}\left(F \times \mathbb{S}^{2} ; H^{2}(F)\right) \cong H_{1}\left(F \times \mathbb{S}^{2} ; \mathbb{Z}\right),
$$

i.e. $H^{3}\left(F \times \mathbb{S}^{2} ; \Pi_{2}(M)\right) \cong H^{3}\left(F \times \mathbb{S}^{2} ; \mathbb{Z}\right)$. Since $F$ is aspherical, $\Pi_{2}\left(F \times \mathbb{S}^{2}\right) \cong \mathbb{Z}$ and so the map $\psi: M \rightarrow F \times \mathbb{S}^{2}$ induces an isomorphism

$$
\psi_{*}: H^{3}\left(F \times \mathbb{S}^{2} ; \Pi_{2}(M)\right) \rightarrow H^{3}\left(F \times \mathbb{S}^{2} ; \Pi_{2}\left(F \times \mathbb{S}^{2}\right)\right) .
$$

By naturality, the image of the obstruction under $\psi_{*}$ is the obstruction to extending $\psi \circ(j \vee \varphi): F \vee \mathbb{S}^{2} \rightarrow F \times \mathbb{S}^{2}$. But the last obstruction vanishes as $\psi \circ(j \vee \varphi)$ is homotopic to the inclusion map (use Corollary 2.4). Therefore $j \vee \varphi$ extends to

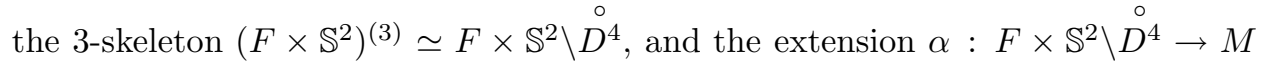
satisfies the property $\psi \circ \alpha \simeq$ inclusion.

Since the map $\psi: M \rightarrow F \times \mathbb{S}^{2}$ has degree one, it induces a splitting of the integral intersection form $\lambda_{M}: H_{2}(M) \times H_{2}(M) \rightarrow \mathbb{Z}$, i.e.

$$
\lambda_{M}=\left(\begin{array}{ll}
0 & 1 \\
1 & 0
\end{array}\right) \oplus \lambda^{\prime} .
$$

By Freedman's theorems (see [4] and [5]) we can realize $\lambda^{\prime}$ as the intersection form of a topological simply-connected 4-manifold $M^{\prime}$, i.e. $\lambda^{\prime}=\lambda_{M^{\prime}}$. Recall that $H_{2}(M ; \Lambda) \cong H_{2}(F) \oplus \operatorname{Ext}_{\Lambda}^{0}\left(H_{2}(M ; \Lambda), \Lambda\right)$, where $Q=\operatorname{Ext}_{\Lambda}^{0}\left(H_{2}(M ; \Lambda), \Lambda\right)$ is stably $\Lambda$-free. Using the universal coefficient spectral sequence

$$
\operatorname{Tor}_{p}^{\Lambda}\left(H_{q}(M ; \Lambda), \mathbb{Z}\right) \Longrightarrow H_{p+q}(M ; \mathbb{Z})
$$

we obtain that

$$
\begin{aligned}
H_{2}(M ; \mathbb{Z}) & \cong \operatorname{Tor}_{0}^{\Lambda}\left(H_{2}(M ; \Lambda), \mathbb{Z}\right) \oplus \operatorname{Tor}_{2}^{\Lambda}\left(H_{0}(M ; \Lambda), \mathbb{Z}\right) \\
& \cong H_{2}(M ; \Lambda) \otimes_{\Lambda} \mathbb{Z} \oplus H_{2}\left(\Pi_{1} ; \mathbb{Z}\right) \\
& \cong\left(H_{2}(F ; \mathbb{Z}) \oplus Q\right) \otimes_{\Lambda} \mathbb{Z} \oplus H_{2}(F ; \mathbb{Z}) \cong \mathbb{Z} \oplus \mathbb{Z} \oplus Q \otimes_{\Lambda} \mathbb{Z} .
\end{aligned}
$$

Note that $Q \otimes_{\Lambda} \mathbb{Z} \cong \oplus_{r} \mathbb{Z}$, where $r=\operatorname{rank} Q$. In particular, we have

$$
Q \otimes_{\Lambda} \mathbb{Z} \cong H_{2}\left(M^{\prime} ; \mathbb{Z}\right)
$$




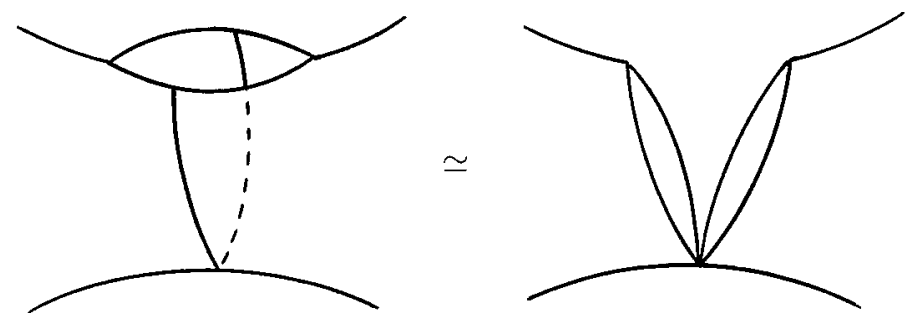

FiguRE 1

and the above decomposition of $H_{2}(M ; \mathbb{Z})$ into a direct sum gives the splitting

$$
\lambda_{M}=\left(\begin{array}{ll}
0 & 1 \\
1 & 0
\end{array}\right) \oplus \lambda_{M^{\prime}}
$$

of the intersection form over $\mathbb{Z}$. In summary, we have

$$
\Pi_{2}(M) \otimes_{\Lambda} \mathbb{Z} \cong(\mathbb{Z} \oplus Q) \otimes_{\Lambda} \mathbb{Z} \cong \mathbb{Z} \oplus H_{2}\left(M^{\prime}\right),
$$

i.e. the $r$ generators of $H_{2}\left(M^{\prime}\right)$ can be represented by maps of 2-spheres. In other words, we have a map $\beta: M^{\prime} \backslash \stackrel{\circ}{D^{4}} \simeq \bigvee_{r} \mathbb{S}^{2} \rightarrow M$. Now we observe that

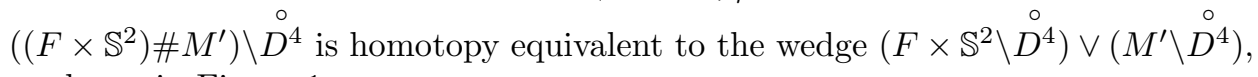
as shown in Figure 1.

Thus the map $\alpha \# \beta \simeq \alpha \vee \beta:\left(F \times \mathbb{S}^{2} \# M^{\prime}\right) \backslash \stackrel{\circ}{D^{4}} \rightarrow M$ induces isomorphisms on $\Pi_{1}$ and on $H_{2}(\cdot ; \mathbb{Z})$. Let us denote $M_{1}=F \times \mathbb{S}^{2} \# M^{\prime}$. The above arguments also imply that the $\Lambda$-ranks of $H_{2}(M ; \Lambda)$ and $H_{2}\left(M_{1} ; \Lambda\right)$ coincide. Next we want to extend $\alpha \# \beta: M_{1} \backslash \stackrel{\circ}{D^{4}} \rightarrow M$ to a map $M_{1} \rightarrow M$. The obstruction for extending $\alpha \# \beta$ is

$$
\theta=\left[\partial\left(M_{1} \backslash \stackrel{\circ}{D^{4}}\right) \stackrel{\alpha \# \beta}{\longrightarrow} M\right] \in \Pi_{3}(M),
$$

i.e. $\theta$ is the homotopy class of the restriction of $\alpha \# \beta$ to the boundary of $M_{1} \backslash \stackrel{\circ}{D^{4}}$.

Obviously, $\theta$ is the image of the generator of

$$
\Pi_{4}\left(M_{1}, M_{1} \backslash \stackrel{\circ}{D^{4}}\right) \cong H_{4}\left(M_{1}, M_{1} \backslash \stackrel{\circ}{D^{4}} ; \Lambda\right) \cong \Lambda
$$

under the composition

$$
\Pi_{4}\left(M_{1}, M_{1} \backslash \stackrel{\circ}{D^{4}}\right) \stackrel{\partial_{*}}{\longrightarrow} \Pi_{3}\left(M_{1} \backslash \stackrel{\circ}{D^{4}}\right) \stackrel{(\alpha \# \beta)_{*}}{\longrightarrow} \Pi_{3}(M) .
$$

Therefore the existence of an extension $h: M_{1} \rightarrow M$ of $\alpha \# \beta$ follows from the following result.

Proposition 2.7. With the above notation, the composition $(\alpha \# \beta)_{*} \circ \partial_{*}$ is the trivial homomorphism.

Using this proposition, we can complete the proof of Theorem 1.1.

Proof of Theorem 1.1. Since the obstruction $\theta$ is zero, there exists a map $h: M_{1} \rightarrow M$ which extends $\alpha \# \beta$. Obviously, $h$ induces an isomorphism on $\Pi_{1}$. It 
suffices to prove that $h_{*}: H_{q}\left(M_{1} ; \Lambda\right) \rightarrow H_{q}(M ; \Lambda)$ is an isomorphism for $q=2,3,4$. Since

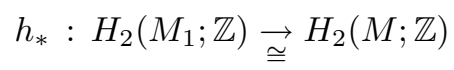

and $H_{2}(M ; \mathbb{Q}) \neq 0$, the map $h$ has degree one if one chooses the appropriate orientations. Hence $h_{*}: H_{q}\left(M_{1} ; \Lambda\right) \rightarrow H_{q}(M ; \Lambda)$ is onto. The kernel $K_{2}(h, \Lambda)$ of $h_{*}: H_{2}\left(M_{1} ; \Lambda\right) \rightarrow H_{2}(M ; \Lambda)$ is $\Lambda$-projective (see [13]); in fact, it is stably $\Lambda$-free. Since the $\Lambda$-ranks of $H_{2}\left(M_{1} ; \Lambda\right)$ and $H_{2}(M ; \Lambda)$ coincide, the $\Lambda$-rank of $K_{2}(h, \Lambda)$ is zero. Therefore $K_{2}(h, \Lambda) \cong 0$, by Kaplansky's lemma (see for example [6] and [8]). By Poincaré duality we obtain isomorphisms for all $q$, i.e. $h$ is a homotopy equivalence, as asserted.

Proof of Proposition 2.7. Note first that $\alpha \# \beta: M_{1} \backslash \stackrel{\circ}{D^{4}} \rightarrow M$ factors over the 3 -skeleton of $M$, i.e.

$$
\alpha \# \beta: M_{1} \backslash \stackrel{\circ}{D^{4}} \rightarrow M \backslash \stackrel{\circ}{D^{4}} \subset M .
$$

Here we have used the identifications $M \backslash \stackrel{\circ}{D^{4}}=M^{(3)}$ and $M_{1} \backslash \stackrel{\circ}{D^{4}}=M_{1}^{(3)}$, where $M^{(q)}$ and $M_{1}^{(q)}$ denote the $q$-skeletons of $M$ and $M_{1}$, respectively. We can also assume that $\alpha \# \beta$ is a cellular map. Consider the following diagram

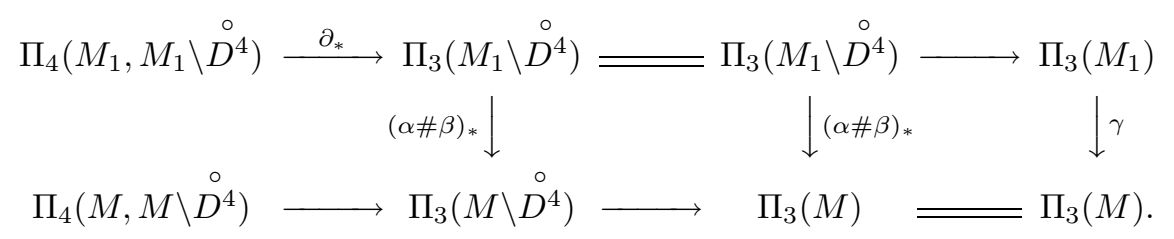

The proof will be completed once we construct a homomorphism

$$
\gamma: \Pi_{3}\left(M_{1}\right) \rightarrow \Pi_{3}(M)
$$

such that the diagram $(/)$ commutes. For this, we consider the Whitehead exact sequence for a 4-dimensional CW-complex $X$ (see [1], [14] and [15]):

$$
H_{4}(\tilde{X}) \longrightarrow \Gamma\left(\Pi_{2}(X)\right) \longrightarrow \Pi_{3}(X) \longrightarrow H_{3}(\tilde{X}) \longrightarrow 0 .
$$

This sequence is natural with respect to maps $X \rightarrow Y$. Here $\tilde{X}$ is the universal covering of $X, \Pi_{3}(X) \rightarrow H_{3}(\widetilde{X})$ is the Hurewicz homomorphism and $\Gamma$ denotes the quadratic functor on abelian groups. We recall that $\Gamma\left(\Pi_{2}(X)\right)$ is equal to $\operatorname{Im}\left(\Pi_{3}\left(X^{(2)}\right) \rightarrow \Pi_{3}\left(X^{(3)}\right)\right)$. In our case, we have $H_{4}(\widetilde{M}) \cong H_{4}\left(\widetilde{M}_{1}\right) \cong 0$ because $\Pi_{1}(M) \cong \Pi_{1}\left(M_{1}\right)$ is an infinite group. Moreover,

$$
H_{3}(\widetilde{M}) \cong H_{3}(M ; \Lambda) \cong H^{1}(M ; \Lambda) \cong H^{1}\left(\Pi_{1} ; \Lambda\right) \cong H^{1}(F ; \Lambda) \cong H_{1}(F ; \Lambda) \cong 0
$$

as $F$ is an aspherical surface. Similarly, $H_{3}\left(\widetilde{M}_{1}\right) \cong 0$. Hence the above sequence implies that $\Gamma\left(\Pi_{2}(M)\right) \cong \Pi_{3}(M)$ and $\Gamma\left(\Pi_{2}\left(M_{1}\right)\right) \cong \Pi_{3}\left(M_{1}\right)$. Now the map $\alpha \# \beta: M_{1} \backslash \stackrel{\circ}{D^{4}} \rightarrow M$ induces $(\alpha \# \beta)_{*}: \Pi_{2}\left(M_{1}\right) \cong \Pi_{2}\left(M_{1} \backslash \stackrel{\circ}{D^{4}}\right) \rightarrow \Pi_{2}(M)$, hence $(\alpha \# \beta)_{* *}: \Gamma\left(\Pi_{2}\left(M_{1}\right)\right) \rightarrow \Gamma\left(\Pi_{2}(M)\right)$. Then the homomorphism $\gamma$ is defined by the 
following diagram:

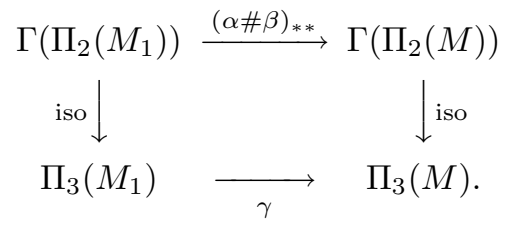

The commutativity of $(/)$ follows from the second interpretation of $\Gamma\left(\Pi_{2}\right)$ looking at the diagram shown below:

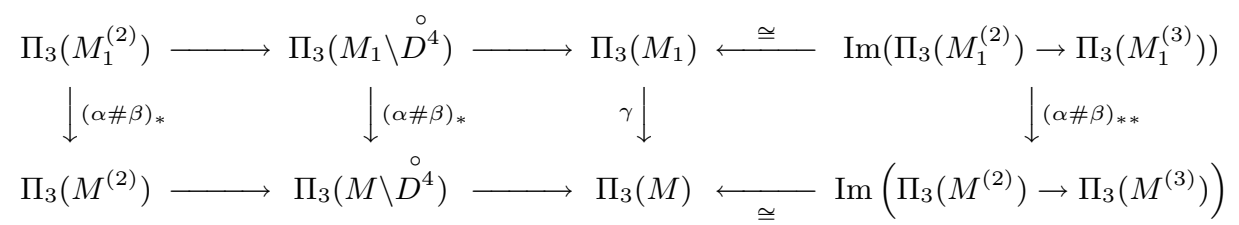

This completes the proof.

Remarks. (1) As a corollary we obtain that in the decomposition $\Pi_{2}(M) \cong \mathbb{Z} \oplus Q$, the $\Lambda$-module $Q$ is actually free. This improves the result of Hillman [6].

(2) The proof of Proposition 2.7 shows that $\gamma: \Pi_{3}\left(M_{1}\right) \rightarrow \Pi_{3}(M)$ is an isomorphism, and hence the sequence

$$
\Pi_{4}\left(M_{1}, M_{1} \backslash \stackrel{\circ}{D^{4}}\right) \stackrel{\partial_{*}}{\longrightarrow} \Pi_{3}\left(M_{1} \backslash \stackrel{\circ}{D^{4}}\right) \stackrel{(\alpha \# \beta)_{*}}{\longrightarrow} \Pi_{3}(M) \longrightarrow 0
$$

is exact.

(3) The proof of Proposition 2.7 can be most easily seen as follows. We write the obstruction $\theta=\theta_{1}+\theta_{2}+\theta_{3}$ according to the splitting

$$
\Pi_{3}(M) \cong \Gamma\left(\Pi_{2}\left(F \times \mathbb{S}^{2}\right)\right) \oplus \Pi_{2}\left(F \times \mathbb{S}^{2}\right) \otimes K_{2}(\psi, \Lambda) \oplus \Gamma\left(K_{2}(\psi, \Lambda)\right)
$$

induced by $H_{2}(M ; \Lambda) \cong H_{2}\left(F \times \mathbb{S}^{2} ; \Lambda\right) \oplus K_{2}(\psi, \Lambda)$.

Now $\theta_{1} \in \Gamma\left(\Pi_{2}\left(F \times \mathbb{S}^{2}\right)\right)$ is zero because it is the obstruction for extending $\psi \circ \alpha$, hence vanishes by Proposition 2.6.

The addendum $\theta_{2} \in \Pi_{2}\left(F \times \mathbb{S}^{2}\right) \otimes K_{2}(\psi, \Lambda)$ is determined by intersection numbers of elements of the submodule $A$, generated by $\operatorname{Im}\left(\alpha_{*}\right) \subset H_{2}(M ; \Lambda)$, and elements of $K_{2}(\psi, \Lambda)$. But they are all zero by construction.

Finally, $\theta_{3} \in \Gamma\left(K_{2}(\psi, \Lambda)\right)$ is zero by hypothesis.

\section{3. $s$-COBORDISM TYPE}

In this section we are going to prove Theorem 1.2. In Section 2 we have constructed a simple homotopy equivalence $h: M \rightarrow F \times \mathbb{S}^{2} \# M^{\prime}$. To obtain Theorem 1.2 , it suffices to prove the following two results.

Proposition 3.1. The pair $(M, h)$ is normally cobordant to a self-homotopy equivalence

$$
g: F \times \mathbb{S}^{2} \# M^{\prime} \rightarrow F \times \mathbb{S}^{2} \# M^{\prime} .
$$

The following is well-known (see [6], Lemma 6, p. 282).

Proposition 3.2. The surgery obstruction map

$$
\theta:\left[\left(F \times \mathbb{S}^{2} \# M^{\prime}\right) \times I,\left(F \times \mathbb{S}^{2} \# M^{\prime}\right) \times \partial I, G / \mathrm{TOP}\right] \rightarrow L_{5}\left(\Pi_{1}\right)
$$

is surjective. 
Now one can use the 5 -dimensional surgery theory to construct an $s$-cobordism between $M$ and $M_{1}=F \times \mathbb{S}^{2} \# M^{\prime}$. In fact, let $W \rightarrow M_{1} \times I$ be a normal cobordism between $(M, h)$ and $\left(M_{1}, g\right)$ guaranteed by Proposition 3.1, i.e. the normal invariants of $(M, h)$ and $\left(M_{1}, g\right)$ coincide. Using the surgery sequence (see [5] and [13]) and Proposition 3.2, it follows that $M_{1}$ and $M$ are topologically s-cobordant. This proves Theorem 1.2.

Since Proposition 3.2 is well-known, it only remains to prove Proposition 3.1. In the case $M^{\prime} \cong \mathbb{S}^{4}$, the result was proved by Hillman (see [7]). To prove Proposition 3.1 we use this result and "paste it together" with the corresponding result for simply-connected topological 4-manifolds (see [3]).

Let us first recall the description of normal invariants (for more details we refer to [2]). Let $\delta: M_{1}=F \times \mathbb{S}^{2} \# M^{\prime} \rightarrow$ BTOP be the classifying map of the stable normal (micro) bundle of $M_{1}$ and let $\rho:$ BTOP $\rightarrow$ BG be the principal fibration with fiber $G$ / TOP. Here BG is the classifying space of stable spherical fibrations, i.e. $\xi=\rho \circ \delta: M_{1} \rightarrow$ BG classifies the Spivak fibration of the Poincaré 4-complex $M_{1}$. Any normal cobordism class of normal maps $N \rightarrow M_{1}$ is determined by a linearization of $\xi$, i.e. by a lifting $\delta^{\prime}$ of $\xi=\rho \circ \delta$

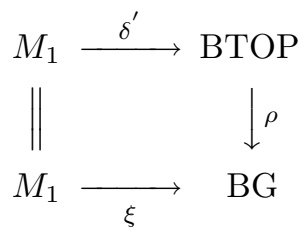

via the Thom construction. This means, fixing the lifting $\delta^{\prime}$, that the normal cobordism classes of normal maps correspond uniquely to the elements of $\left[M_{1}, G / \mathrm{TOP}\right]$, i.e. $\delta^{\prime}(x)=g(x) \delta(x)$ with $g: M_{1} \rightarrow G /$ TOP. Let $\Sigma^{3} \subset M_{1}=F \times \mathbb{S}^{2} \# M^{\prime}$ be the 3 -sphere along which the manifolds $F \times \mathbb{S}^{2}$ and $M^{\prime}$ are glued together. Then

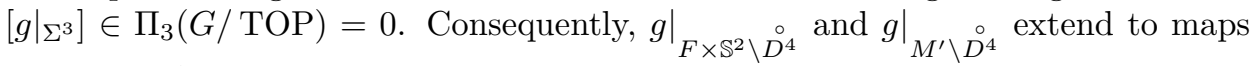
$g_{1}: F \times \mathbb{S}^{2} \rightarrow G /$ TOP and $g_{2}: M^{\prime} \rightarrow G /$ TOP, respectively. The values of $g_{1}$ and $g_{2}$ coincide on the 4 -ball $D^{4}$. Two extensions of $\left.g\right|_{\Sigma^{3}}$ over the 4 -ball $D^{4}$ differ by an element of $\Pi_{4}(G / \mathrm{TOP}) \cong \mathbb{Z}$. We use the unique extension of $\left.g\right|_{\Sigma^{3}}$ such that the surgery obstruction of $g_{2}$ is zero. In other words, we have constructed a map

$$
\mu:\left[F \times \mathbb{S}^{2} \# M^{\prime}, G / \mathrm{TOP}\right] \rightarrow\left[F \times \mathbb{S}^{2}, G / \mathrm{TOP}\right] \oplus\left[M^{\prime}, G / \mathrm{TOP}\right]
$$

which sends $[g]$ into $\left(\left[g_{1}\right],\left[g_{2}\right]\right)$.

On the other hand, attaching a 4 -ball $D^{4}$ to $\Sigma^{3}$ yields a map

$$
t: F \times \mathbb{S}^{2} \# M^{\prime} \rightarrow F \times \mathbb{S}^{2} \# M^{\prime} \cup_{\Sigma^{3}} D^{4} \simeq F \times \mathbb{S}^{2} \vee M^{\prime}
$$

which induces

$$
\begin{aligned}
t_{*}:\left[F \times \mathbb{S}^{2} \vee M^{\prime}, G / \mathrm{TOP}\right] & \cong\left[F \times \mathbb{S}^{2}, G / \mathrm{TOP}\right] \oplus\left[M^{\prime}, G / \mathrm{TOP}\right] \\
& \rightarrow\left[F \times \mathbb{S}^{2} \# M^{\prime}, G / \mathrm{TOP}\right] .
\end{aligned}
$$

Now it is very easy to see that $t_{*} \circ \mu$ is the identity, hence $t_{*}$ is surjective. On the other hand, the connected sum with $\left(M^{\prime}, g_{2}\right)$ gives the following commutative 
diagram

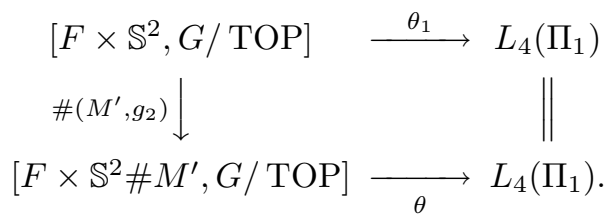

The map induced on $L_{4}\left(\Pi_{1}\right)$ is the identity because the surgery obstruction of $\left(M^{\prime}, g_{2}\right)$ is zero. If $g: F \times \mathbb{S}^{2} \# M^{\prime} \rightarrow G /$ TOP is the normal invariant of a given (simple) homotopy equivalence $h: M \rightarrow F \times \mathbb{S}^{2} \# M^{\prime}$ and $\mu([g])=\left(\left[g_{1}\right],\left[g_{2}\right]\right)$, then $\theta_{1}\left(g_{1}\right)=0$. This follows from the diagram $(/ /)$ and the fact that $\theta_{2}\left(g_{2}\right)=0$, where $\theta_{2}:\left[M^{\prime}, G / \mathrm{TOP}\right] \rightarrow L_{4}(1)$.

In summary, we have proved the following result.

Proposition 3.3. Any element $[g] \in\left[F \times \mathbb{S}^{2} \# M^{\prime}, G / \mathrm{TOP}\right]$, coming from a (simple) homotopy equivalence $h: M \rightarrow F \times \mathbb{S}^{2} \# M^{\prime}$, belongs to $\operatorname{Im} t_{*}$.

More precisely, there are elements

$$
\begin{aligned}
& {\left[g_{1}\right] \in \operatorname{Ker}\left(\theta_{1}:\left[F \times \mathbb{S}^{2}, G / \mathrm{TOP}\right] \rightarrow L_{4}\left(\Pi_{1}\right)\right),} \\
& {\left[g_{2}\right] \in \operatorname{Ker}\left(\theta_{2}:\left[M^{\prime}, G / \mathrm{TOP}\right] \rightarrow L_{4}(1)\right)}
\end{aligned}
$$

such that $t_{*}\left(\left[g_{1}\right],\left[g_{2}\right]\right)=[g]$.

To finish the proof of Proposition 3.1 we recall that the elements of $\operatorname{Ker}\left(\theta_{1}\right)$ and $\operatorname{Ker}\left(\theta_{2}\right)$ come from elements of $\operatorname{HE}_{\mathrm{Id}}\left(F \times \mathbb{S}^{2}\right)$ and $\operatorname{HE}_{\mathrm{Id}}\left(M^{\prime}\right)$, respectively (see [3] and [7]). Here $\mathrm{HE}_{\mathrm{Id}}$ denotes the set of homotopy classes of simple self-homotopy equivalences inducing the identities on $\Pi_{1}$ and on $H_{*}$. More precisely, the proofs of the results of the quoted papers show that there are representatives in $\mathrm{HE}_{\mathrm{Id}}$ leaving a 4-ball fixed. Therefore, if $h_{1}: F \times \mathbb{S}^{2} \rightarrow F \times \mathbb{S}^{2}$ and $h_{2}: M^{\prime} \rightarrow M^{\prime}$ are such representatives of $g_{1}$ and $g_{2}$, then $\left.h_{i}\right|_{D_{i}^{4}}=$ identity for $i=1,2$. Thus we can form the map $h_{1} \# h_{2}: M_{1} \rightarrow M_{1}$. Obviously, $h$ and $h_{1} \# h_{2}$ have the same normal invariants. This proves Proposition 3.1.

In this section we did not use the hypothesis that $w_{2}(M)=0$. In fact, our arguments prove the following more general result.

Theorem 3.4. Let $M^{4}$ be a closed connected oriented (TOP) 4-manifold homotopy equivalent to $E \# M^{\prime}$, where $E$ is an $\mathbb{S}^{2}$-bundle over a closed oriented aspherical surface $F$ and $M^{\prime}$ is a simply-connected 4-manifold. Then $M$ is topologically scobordant to $E \# M^{\prime}$.

\section{Appendix}

As announced in the introduction, here we will treat the case $w_{2}(u) \neq 0$. First recall that there is only one twisted $\mathbb{S}^{2}$-bundle over an oriented closed surface $F$, denoted by $F \underset{\sim}{\times} \mathbb{S}^{2}$, because these bundles are determined by the first and second

Stiefel-Whitney classes. It can be obtained from $\left(F \backslash \stackrel{\circ}{D^{2}}\right) \times \mathbb{S}^{2}$ by attaching $D^{2} \times \mathbb{S}^{2}$ with a map $\alpha: \partial D^{2} \times \mathbb{S}^{2} \rightarrow \partial D^{2} \times \mathbb{S}^{2}$ associated to the generator of $\Pi_{1}(\mathrm{SO}(3))$. The intersection matrix of $F \times \mathbb{S}^{2}$ is

$$
\left(\begin{array}{ll}
1 & 1 \\
1 & 0
\end{array}\right)
$$


with respect to $x, y \in H_{2}\left(F \times \mathbb{S}^{2}\right)$, where $y$ represents the fiber and $\pi_{*}(x)=[F]$, $\pi: F \underset{\sim}{\sim} \mathbb{S}^{2} \rightarrow F$ being the fiber projection.

Proposition 4.1. Let $M^{4}$ be a closed connected oriented 4-manifold with $\Pi_{1}(M) \cong$ $\Pi_{1}(F)$. Assume that $w_{2}(u) \neq 0$ (notation as in Section 2). Then there is a map

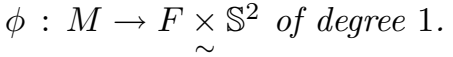

Proof. Let $f: M \rightarrow F$ and $g^{\prime}: M \rightarrow \mathbb{C} P^{\infty}$ be as in the proof of Lemma 2.5. Then the restriction

$$
f \times\left. g^{\prime}\right|_{M \backslash D^{4}}: M \backslash \stackrel{\circ}{D^{4}} \rightarrow F \times \mathbb{C} P^{\infty}
$$

factors as follows:

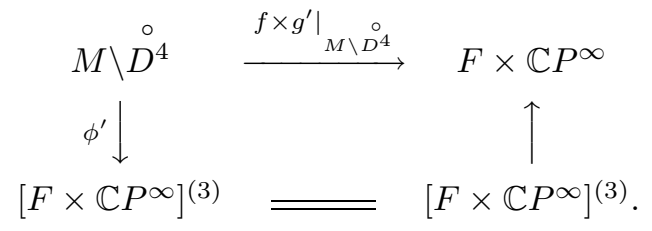

But note that $\left[F \times \mathbb{C} P^{\infty}\right]^{(3)}=F \times \mathbb{S}^{2} \backslash \stackrel{\circ}{B^{4}}, B^{4}$ being a 4-ball. Hence we have a map

$$
\phi^{\prime}: M \backslash \stackrel{\circ}{D^{4}} \rightarrow F \times \mathbb{S}^{2} \backslash \stackrel{\circ}{B^{4}} \text {. }
$$

Obviously $\phi^{\prime}$ extends to $\phi: M \rightarrow\left(F \times \mathbb{S}^{2} \backslash \stackrel{\circ}{B^{4}}\right) \cup_{\lambda} D^{4}$, where $\lambda=\left.\phi^{\prime}\right|_{\partial D^{4}}$. Therefore

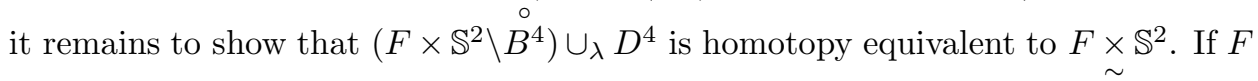
were $\mathbb{S}^{2}$, then $\left(F \times \mathbb{S}^{2} \backslash \stackrel{\circ}{B^{4}}\right) \cup_{\lambda} D^{4}$ is a Poincaré complex with intersection matrix

$$
\left(\begin{array}{ll}
1 & 1 \\
1 & 0
\end{array}\right)
$$

It is homotopy equivalent to $\mathbb{S}^{2} \times \mathbb{S}^{2}$. The general case can be reduced to that of a sphere $\mathbb{S}^{2}$ by considering the collapsing map

$$
c: F \underset{\sim}{\sim} \mathbb{S}^{2} \rightarrow\left(F /\left(F \backslash \stackrel{\circ}{D_{1}^{2}}\right)\right) \underset{\sim}{\times} \mathbb{S}^{2} \cong \mathbb{S}^{2} \underset{\sim}{\sim} \mathbb{S}^{2} .
$$

Here $D_{1}^{2} \subset F$ is a 2-disc which contains in its interior the 2-disc $D^{2} \subset F$ used at the beginning of the section to describe

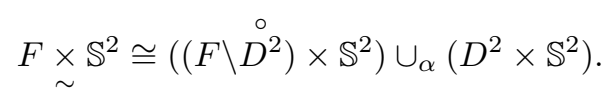

The general case will follow from the fact that $\phi^{\prime}$ can be homotoped in a collar of the boundary of $M \backslash \stackrel{\circ}{D^{4}}$ such that $\phi^{\prime}\left(\partial D^{4}\right) \subset \stackrel{\circ}{D_{1}^{2}} \times \mathbb{S}^{2}$. To extend $\left.\phi^{\prime}\right|_{\partial D^{4}}$ over $D^{4}$ we need to reglue $\stackrel{\circ}{D^{2}} \times \mathbb{S}^{2} \subset D_{1}^{2} \times \mathbb{S}^{2}$ by the twist $\alpha: \partial D^{2} \times \mathbb{S}^{2} \rightarrow \partial D^{2} \times \mathbb{S}^{2}$, i.e. we have to form

$$
F \underset{\sim}{\times} \mathbb{S}^{2} \cong\left(\left(F \backslash \stackrel{\circ}{D^{2}}\right) \times \mathbb{S}^{2}\right) \cup_{\alpha}\left(D^{2} \times \mathbb{S}^{2}\right)
$$


To see that we can assume $\phi^{\prime}\left(\partial D^{4}\right) \subset \stackrel{\circ}{D_{1}^{2}} \times \mathbb{S}^{2}$ we consider the short exact homotopy sequence (recall that $F$ is now aspherical, so $\Pi_{3}\left(F \times \mathbb{S}^{2}\right) \cong \mathbb{Z}$ ):

$$
0 \rightarrow \Pi_{4}\left(F \times \mathbb{S}^{2}, F \times \mathbb{S}^{2} \backslash \stackrel{\circ}{B^{4}}\right) \cong \Lambda \rightarrow \Pi_{3}\left(F \times \mathbb{S}^{2} \backslash \stackrel{\circ}{B^{4}}\right) \rightarrow \Pi_{3}\left(F \times \mathbb{S}^{2}\right) \cong \mathbb{Z} \rightarrow 0 .
$$

This sequence splits because

$$
\operatorname{Ext}_{\Lambda}^{1}(\mathbb{Z}, \Lambda) \cong H^{1}(F ; \Lambda) \cong H_{1}(F ; \Lambda) \cong 0 .
$$

Then we have $[\lambda]=\left[\lambda_{1}\right]+\left[\lambda_{2}\right] \in \Lambda \oplus \mathbb{Z}$. Therefore $\left[\lambda_{2}\right]=k[\eta]$, where $k \in \mathbb{Z}$, $\eta: \mathbb{S}^{3} \rightarrow\{*\} \times \mathbb{S}^{2}$ is the Hopf map and $* \in \stackrel{\circ}{D_{1}^{2}}$. It follows that $\lambda_{2}\left(\partial \stackrel{\circ}{D^{4}}\right) \subset \stackrel{\circ}{D_{1}^{2}} \times \mathbb{S}^{2}$. On the other hand we choose $B^{4}=D^{2} \times D_{-}^{2} \subset F \times \mathbb{S}^{2}$, where $D_{-}^{2}$ is the lower hemisphere. Hence a generator $\tau$ of $\Lambda \subset \Pi_{3}\left(F \times \mathbb{S}^{2} \backslash \stackrel{\circ}{B^{4}}\right)$ has image in $D_{1}^{2} \times \mathbb{S}^{2}$. Since $\left[\lambda_{1}\right]=a \tau$, where $a \in \Lambda$, the image of $\lambda_{1}$ belongs to $D_{1}^{2} \times \mathbb{S}^{2}$, up to some arcs running through $\left(F \backslash \stackrel{\circ}{D^{2}}\right) \times \mathbb{S}^{2}$. This completes the proof.

Since the other arguments are the same as in the case $w_{2}(u)=0$, we have completed Therorem 1.1 with the following result involving twisted $\mathbb{S}^{2}$-bundles over aspherical surfaces.

Theorem 1.1'. Let $M^{4}$ be a closed connected oriented 4-manifold with $\Pi_{1}(M) \cong$ $\Pi_{1}(F)$. Assume that $w_{2}(u) \neq 0$ (notation as in Section 2). Then $M$ is simple homotopy equivalent to the connected sum $M_{1}=\left(F \times \mathbb{S}^{2}\right) \# M^{\prime}$, where $M^{\prime}$ is the simply-connected 4-manifold defined in the discussion preceding the statement of Theorem 1.1, if and only if the $\Lambda$-intersection forms on $K_{2}(\phi, \Lambda)$ and on $K_{2}\left(c^{\prime}, \Lambda\right)$ are isomorphic, where $c^{\prime}$ denotes the collapsing map from $M_{1}$ to $F \times \mathbb{S}^{2}$. Moreover, the manifolds $M$ and $\left(F \times \mathbb{S}^{2}\right) \# M^{\prime}$ are topologically s-cobordant.

\section{REFERENCES}

1. H. J. Baues, Combinatorial Homotopy and 4-dimensional Complexes, Walter de Gruyter, Berlin - New York, 1991. MR 92h:55008

2. W. Browder, Surgery on Simply-connected Manifolds, Springer-Verlag, Berlin- HeidelbergNew York, 1972. MR 50:11272

3. T.D. Cochran and N. Habegger, On the homotopy theory of simply-connected four-manifolds, Topology 29 (1990), 419-440. MR 91h:57006

4. M.H. Freedman, The topology of four-dimensional manifolds, J. Differential Geom. 17 (1982), 357-453. MR 84b:57006

5. M.H. Freedman and F. Quinn, Topology of 4-manifolds, Princeton Univ. Press, Princeton, New Jersey, 1990. MR 94b:57021

6. J.A. Hillman, On 4-manifolds homotopy equivalent to surface bundles over surfaces, Topology Appl. 40 (1991), 275-286. MR 92g:57031

7. J.A. Hillman, On 4-manifolds with universal covering spaces $\mathbb{S}^{2} \times \mathbb{R}^{2}$ or $\mathbb{S}^{3} \times \mathbb{R}$, Topology Appl. 52 (1993), 23-42. MR 95b:57020

8. J.A. Hillman, The Algebraic Characterization of Geometric 4-Manifolds, London Math. Soc. Lect. Note Ser. 198, Cambridge Univ. Press, Cambridge, 1994. MR 95m:57032

9. Y. Matsumoto, Personal Communication, 1995.

10. Y. Matsumoto, Diffeomorphism types of elliptic surfaces, Topology 25 (1986), 549-563. MR 88b:32061

11. J. Milnor, Whitehead torsion, Bull. Amer. Math. Soc. 72 (1966), 358-426. MR 33:4922

12. C.T.C. Wall, On simply-connected 4-manifolds, J. London Math. Soc. 39 (1964), 141-149. MR 29:627

13. C.T.C. Wall, Surgery on Compact Manifolds, Academic Press, London-New York, 1970. MR 55:4217 
14. J.H.C. Whitehead, A certain exact sequence, Ann. of Math. (2) 52 (1950), 51-110. MR 12:43c

15. J.H.C. Whitehead, Elements of Homotopy Theory, Springer-Verlag, Berlin- Heidelberg- New York, 1978. MR 80b:55001

Dipartimento di Matematica, Università di Modena, Via Campi 213/B, 41100 Modena, ITALY

E-mail address: albertoc@unimo.it

Dipartimento di Matematica, Università di Milano, Via Saldini 50, 20133 Milano, ITALY

E-mail address: hegenbarth@vmimat.mat.unimi.it

Institute for Mathematics, Physics and Mechanics, University of Ljubljana, P. O. Box 64, Luubluana 61111, Slovenia

E-mail address: Dusan.repovs@uni-lj.si 